Article

\title{
Photo-Thermal Interactions in a Semiconducting Media with a Spherical Cavity under Hyperbolic Two-Temperature Model
}

\author{
Faris S. Alzahrani ${ }^{1}$ and Ibrahim A. Abbas ${ }^{1,2, *}$ \\ 1 Nonlinear Analysis and Applied Mathematics Research Group (NAAM), Department of Mathematics, \\ King Abdulaziz University, Jeddah 21521, Saudi Arabia; falzahrani1@kau.edu.sa \\ 2 Department of Mathematics, Faculty of Science, Sohag University, Sohag 82524, Egypt \\ * Correspondence: ibrabbas7@science.sohag.edu.eg
}

Received: 23 March 2020; Accepted: 8 April 2020; Published: 15 April 2020

check for updates

\begin{abstract}
This article highlights the study of photo-thermoelastic interaction in an unbounded semiconductor medium containing a spherical cavity. This problem is solved using the new hyperbolic two-temperature model. The bounding surface of the cavity is traction free and loaded thermally by exponentially decaying pulse boundary heat flux. In addition, the carrier density is prescribed on the inner surface of the cavity in terms of the recombination speed. The techniques of Laplace transforms are used to get the analytical solutions of the problem in the transformed domain by the eigenvalues method. The inversions of Laplace transformations have been carried out numerically. The outcomes also display that the analytical schemes can overcome the mathematical problem to analyze this problem. Numerical outcomes for a semiconductor material are performed and demonstrated graphically. According to the numerical results, this new hyperbolic two-temperature model of thermoelasticity offers finite speed of the thermal wave and mechanical wave propagation.
\end{abstract}

Keywords: Laplace transforms; hyperbolic two-temperature; spherical cavity; eigenvalues method; semiconductor medium

\section{Introduction}

The thermoelasticity model with the classical two-temperature model is presented by Williams and Gurtin [1], Chen and Gurtin [2] and Chen et al. [3]; when using another temperature depending on the classic two temperatures (thermodynamic temperature $T^{*}$ and conductivity temperature $\phi^{*}$ ), the classical inequality of Clausius-Duhem has been substituted. The first is due to the thermal processes and the second is due to the inherent mechanical processes between the particles and the elastic material layers. Only in the last decade were these processes observed, developed in many works in the theory of thermoelasticity under two temperatures, and applications found mainly in problems in which the discontinuities of restrictions have no physical interpretations. Ezzat et al. [4] investigated the effects of fractional order and two-temperature theory in magneto-thermoelasticity under dual-phase-lag heat transfer. Ezzat and Youssef [5] studied the two-temperature model as a three-dimensional problem for a thermoelastic plane due to ramp type heating. Deswal and Kalkal [6] have used the state space formulations to study the impacts of initial stresses under two-temperature magneto-thermoelasticity. Abbas et al. [7] have studied the response of a heating source in a transversely isotropic thermoelastic medium under two temperatures without energy dissipation. Youssef and El-Bary [8] have presented new generalized thermoelastic interactions under the hyperbolic two-temperature model.

In integrated circuit fabrication, silicon is normally used as the basis material and produces an N-type or P-type semiconductor by adopting diffusion technology. On applying the thermal effects, the surface of steel parts can be carburized by applying the diffusion principle. In the electronics 
domain, the diffusion movement is also utilized in forming a semiconductor PN junction with free electrons and holes. The understanding of transport phenomena in solids through the expansions of spatially resolved in situ investigations has gained major attention. This paper describes investigations that try to measure the transport process based on the principles of optical beams deviation through a photothermal technique that can be considered as an expansion of the photothermal deviation approach. These techniques are characterized as having no contact and directly producing the parameters of electronics and heat transport on the surface of the semiconductor or at the interface and within most of the semiconducting material. Marin et al. $[9,10]$ have investigated some problems for elastic dipolar bodies.

Pure silicon is an intrinsic semiconductor and is used in a wide range of the semiconductor industry, for instance, for monocrystal lines $S i$ is used to produce silicon wafers. Todorovic [11,12] has studied the plasma and thermal-elastic waves in semiconductor material. The change in the propagation of thermal, elastic and plasma waves are due to linear coupling through heat and mass transport (i.e., thermal-diffusive). In materials science, the heating conductivity variables that depend on temperature are very significant and have numerous applications. Song et al. [13] studied vibration using the generalized thermoelastic model subjected to optically excited semiconductors as micro-conductors. Yasein et al. [14] studied the influence of variable thermal conductivity of one-dimensional semiconducting media under photothermal excitations due to ramp type heating. Ailawalia and Kumar [15] applied ramp type heating to study the semiconducting media under the photothermal theory. Abbas and Hobiny [16] studied the photo-thermal-elastic interaction in an unbounded semiconducting medium with spherical cavity, due to pulse heat flux, by using the theory of coupled plasma, thermal, and elastic wave. Yasein, et al. [14] studied the influence of variable thermal conductivity of a semiconductor elastic medium during photothermal excitation subjected to thermal ramp type. Lotfy et al. [17] investigated the response of a semiconductor medium of variable thermal conductivity due to laser pulses with two temperatures through the photothermal process. Many researchers [18-26] have solved various problems under generalized thermoelastic models. In the Laplace domain, the eigenvalues approach gave an analytical solution without any supposed restrictions on the factual physical variables.

The main objective of the present contribution is to study the effects of hyperbolic two-temperature in a semiconductor medium with a spherical cavity under pulse heat flux. The basic equations are processed by using the eigenvalues method and Laplace transforms. Employing Laplace transform as tools, the solutions of the thermo-physical quantities have been obtained in the transformed domain, and the numerical inversion of the Laplace transform is carried out incorporating a method proposed by Stehfest. Finally, the outcomes are illustrated graphically to show the differences among the hyperbolic two-temperature model, the classical two-temperature model, and the one temperature model.

\section{Formulation of the Problem}

The basic governing equations under the hyperbolic two-temperature photothermal theory for a homogeneous, isotropic semiconductor medium in the absence of body forces and heat sources are given by $[8,27,28]$ :

$$
\begin{gathered}
\mu u_{i, j j}+(\lambda+\mu) u_{j, i j}-\gamma_{n} N_{, i}-\gamma_{t} T_{, i}=\rho \frac{\partial^{2} u_{i}}{\partial t^{2}}, \\
D_{e} N_{, j j}-\frac{N}{\tau}+\frac{k}{\tau} T=\frac{\partial N}{\partial t}, \\
K \phi_{, j j}+\frac{E_{g}}{\tau} N=\rho c_{e} \frac{\partial T}{\partial t}+\gamma_{t} T_{o} \frac{\partial u_{j, j}}{\partial t}, \\
\ddot{\phi}-\ddot{T}=a \phi_{, j j},
\end{gathered}
$$

where $i, j, k=1,2,3, N=n-n_{o}, n_{o}$ is the concentration of carrier at equilibrium, $u_{i}$ are the components of displacement, $T=T^{*}-T_{o}, T^{*}$ is the thermodynamic temperature increment, $\phi=\phi^{*}-T_{0}, \varphi^{*}$ is the 
temperature conductivity increment, $\rho$ is the medium density, $T_{o}$ is the reference temperature, $a>0$ is the parameter of two-temperature, $\lambda, \mu$ are the Lame's constants, $\sigma_{i j}$ are the components of stresses, $c_{e}$ is the specific heat at constant strain, $\gamma_{n}=(3 \lambda+2 \mu) d_{n}, d_{n}$ is the coefficient of electronic deformation, $\gamma_{t}=(3 \lambda+2 \mu) \alpha_{t}, \alpha_{t}$ is the linear thermal expansion coefficient, $K$ is the thermal conductivity, $\tau$ is the lifetime of a photogenerated carrier, $D_{e}$ is the coefficient of carrier diffusion, $k=\frac{\partial n_{o}}{\partial T}$ is the coupling parameter of thermal activation [28], $r$ is the position vector, and $t$ is the time. Let us consider an isotropic, homogeneous unbounded semiconductor medium with a spherical cavity, whose state can be expressed in terms of the time $t$ and the space variable $r$ which occupies the region $R \leq r<\infty$. Due to symmetry, the radial displacement $u_{r}=u(r, t)$ is only non-vanishing, therefore the Equations (1)-(4) can be written as:

$$
\begin{gathered}
(\lambda+2 \mu)\left(\frac{\partial^{2} u}{\partial r^{2}}+\frac{2}{r} \frac{\partial u}{\partial r}-\frac{u}{r^{2}}\right)-\gamma_{n} \frac{\partial N}{\partial r}-\gamma_{t} \frac{\partial T}{\partial r}=\rho \frac{\partial^{2} u}{\partial t^{2}} \\
D_{e}\left(\frac{\partial^{2} N}{\partial r^{2}}+\frac{2}{r} \frac{\partial N}{\partial r}\right)=\frac{\partial N}{\partial t}+\frac{N}{\tau}-\frac{k}{\tau} T, \\
K\left(\frac{\partial^{2} \phi}{\partial r^{2}}+\frac{2}{r} \frac{\partial \phi}{\partial r}\right)=\rho c_{e} \frac{\partial T}{\partial t}-\frac{E_{g}}{\tau} N+\gamma_{t} T_{o} \frac{\partial}{\partial t}\left(\frac{\partial u}{\partial r}+\frac{2 u}{r}\right), \\
\frac{\partial^{2} T}{\partial t^{2}}=\frac{\partial^{2} \phi}{\partial t^{2}}-a\left(\frac{\partial^{2} \phi}{\partial r^{2}}+\frac{2}{r} \frac{\partial \phi}{\partial r}\right),
\end{gathered}
$$

with

$$
\begin{gathered}
\sigma_{r r}=(\lambda+2 \mu) \frac{\partial u}{\partial r}+\lambda \frac{2 u}{r}-\gamma_{n} N-\gamma_{t} T \text { and } \\
\sigma_{\theta \theta}=\sigma_{\phi \phi}=(\lambda+2 \mu) \frac{u}{r}+\lambda\left(\frac{\partial u}{\partial r}+\frac{u}{r}\right)-\gamma_{n} N-\gamma_{t} T .
\end{gathered}
$$

\section{Initial and Boundary Conditions}

Take into account the boundary with initial conditions before solving the problem. In this problem, the initial conditions are assumed to be homogeneous and are supplemented by considering the boundary on the internal surface of the cavity. It is stress free, therefore the boundary condition can be given as:

$$
\sigma_{r r}(R, t)=0
$$

The conditions on the inner surface of the cavity are due to heat flux with an exponentially decaying pulse [29]

$$
-\left.K \frac{\partial \phi(r, t)}{\partial r}\right|_{r=R}=q_{o} \frac{t^{2} e^{-\frac{t}{t_{p}}}}{16 t_{p}^{2}}
$$

where $t_{p}$ is the pulsing heat flux characteristic time and $q_{o}$ is a constant. The carrier density boundary condition on the inner surface of the cavity may be expressed by

$$
\left.D_{e} \frac{\partial N(r, t)}{\partial r}\right|_{r=R}=s_{a} N(R, t),
$$

where $s_{O}$ is the speed of recombination on the surface. For appropriateness, the non-dimensional parameters can be taken as

$$
\begin{gathered}
N^{\circ}=\frac{N}{n_{o}}, \phi^{\circ}=\frac{\phi}{T_{o}}, T^{\circ}=\frac{T}{T_{o}},\left(r^{\circ}, u^{\circ}\right)=\eta c(r, u),\left(\sigma_{r r}^{\circ}, \sigma_{\theta \theta}^{\circ}\right)=\frac{\left(\sigma_{r r}, \sigma_{\theta \theta}\right)}{\lambda+2 \mu} \\
\left(t^{\circ}, \tau^{\circ}, t_{p}^{\circ}\right)=\eta c^{2}\left(t, \tau, t_{p}\right), q_{o}^{\circ}=\frac{q_{o}}{\eta c T_{0} K}, a^{\circ}=\frac{a}{c^{2}}
\end{gathered}
$$


where $\eta=\frac{\rho c_{e}}{K}$ and $c^{2}=\frac{\lambda+2 \mu}{\rho}$.

In these dimensionless terms of the physical quantities in Equation (14), the above Equations (6)-(14) can be expressed as (after neglecting the superscript ${ }^{\circ}$ for convenience)

$$
\begin{gathered}
\frac{\partial^{2} u}{\partial r^{2}}+\frac{2}{r} \frac{\partial u}{\partial r}-\frac{2 u}{r^{2}}-n_{1} \frac{\partial N}{\partial r}-n_{2} \frac{\partial T}{\partial r}=\frac{\partial^{2} u}{\partial t^{2}}, \\
\frac{\partial^{2} N}{\partial r^{2}}+\frac{2}{r} \frac{\partial N}{\partial r}=n_{3} \frac{\partial N}{\partial t}+\frac{n_{3}}{\tau} N-\frac{n_{4}}{\tau} T, \\
\frac{\partial^{2} \phi}{\partial r^{2}}+\frac{2}{r} \frac{\partial \phi}{\partial r}=\frac{\partial T}{\partial t}-\frac{n_{5}}{\tau} N+v_{6} \frac{\partial}{\partial t}\left(\frac{\partial u}{\partial r}+\frac{2 u}{r}\right) . \\
\frac{\partial^{2} T}{\partial t^{2}}=\frac{\partial^{2} \phi}{\partial t^{2}}-a\left(\frac{\partial^{2} \phi}{\partial r^{2}}+\frac{2}{r} \frac{\partial \phi}{\partial r}\right), \\
\sigma_{r r}=\frac{\partial u}{\partial r}+n_{7} \frac{2 u}{r}-n_{1} N-n_{2} T, \\
\sigma_{\theta \theta}=n_{7} \frac{\partial u}{\partial r}+\left(1+n_{7}\right) \frac{u}{r}-n_{1} N-n_{2} T, \\
=-q_{o} \frac{t^{2} e^{-\frac{t}{t_{p}}}}{16 t_{p}^{2}}, \sigma_{r r}(R, t)=0,\left.\frac{\partial N(r, t)}{\partial r}\right|_{r=R}=f_{8} N(R, t),
\end{gathered}
$$

where $n_{1}=\frac{n_{o} \gamma_{n}}{\lambda+2 \mu}, n_{2}=\frac{T_{o} \gamma_{t}}{\lambda+2 \mu}, n_{3}=\frac{1}{\eta D_{e}}, n_{4}=\frac{k T_{o}}{n_{o} \eta D_{e}}, n_{5}=\frac{n_{o} E_{g}}{\rho c_{e} T_{0}}, n_{6}=\frac{\gamma_{t}}{\rho c_{e}}, n_{7}=\frac{\lambda}{\lambda+2 \mu}, n_{8}=\frac{S_{a}}{\eta c D_{e}}$.

\section{Method of Solution}

For the function $\mathrm{h}(r, t)$, the Laplace transform is defined by

$$
\overline{\mathrm{h}}(r, s)=L[\mathrm{~h}(r, t)]=\int_{0}^{\infty} \mathrm{h}(r, t) e^{-s t} d t, s>0,
$$

where $s$ is the parameter of the Laplace transform. Hence, the above equations can be written in the following forms

$$
\begin{aligned}
& \frac{d^{2} \bar{u}}{d r^{2}}+\frac{2}{r} \frac{d \bar{u}}{d r}-\frac{2 \bar{u}}{r^{2}}-n_{1} \frac{d \bar{N}}{d r}-n_{2} \frac{d \bar{T}}{d r}=s^{2} \bar{u}, \\
& \frac{d^{2} \bar{N}}{d r^{2}}+\frac{2}{r} \frac{d \bar{N}}{d r}=n_{3}\left(s+\frac{1}{\tau}\right) \bar{N}-\frac{n_{4}}{\tau} \bar{T}, \\
& \frac{d^{2} \bar{\phi}}{d r^{2}}+\frac{2}{r} \frac{d \bar{\phi}}{d r}=s \bar{T}-\frac{n_{5}}{\tau} \bar{N}+s n_{6}\left(\frac{d \bar{u}}{d r}+\frac{2 \bar{u}}{r}\right), \\
& \bar{T}=\bar{\phi}-\frac{a}{s^{2}}\left(\frac{d^{2} \bar{\phi}}{d r^{2}}+\frac{2}{r} \frac{d \bar{\phi}}{d r}\right), \\
& \bar{\sigma}_{r r}=\frac{d \bar{u}}{d r}+n_{7} \frac{2 \bar{u}}{r}-n_{1} \bar{N}-n_{2} \bar{T}, \\
& \bar{\sigma}_{\theta \theta}=n_{7} \frac{d \bar{u}}{d r}+\left(1+n_{7}\right) \frac{\bar{u}}{r}-n_{1} \bar{N}-n_{2} \bar{T}, \\
& \left.\frac{d \bar{\phi}(r, t)}{d r}\right|_{r=R}=\frac{-q_{o} t_{p}}{8\left(s t_{p}+1\right)^{3}}, \bar{\sigma}_{r r}(R, r)=0,\left.\frac{d \bar{N}(r, t)}{d r}\right|_{r=R}=n_{8} \bar{N}(R, t) .
\end{aligned}
$$


Differentiating Equations (23)-(25) with respect to $r$ and using Equation (26), which can be written as

$$
\begin{gathered}
\frac{d^{2} \bar{u}}{d r^{2}}+\frac{2}{r} \frac{d \bar{u}}{d r}-\frac{2 \bar{u}}{r^{2}}=a_{1} \bar{u}+a_{2} \frac{d \bar{N}}{d r}+a_{3} \frac{d \bar{\phi}}{d r} \\
\frac{d^{2}}{d r^{2}}\left(\frac{d \bar{N}}{d r}\right)+\frac{2}{r} \frac{d}{d r}\left(\frac{d \bar{N}}{d r}\right)-\frac{2}{r^{2}}\left(\frac{d \bar{N}}{d r}\right)=a_{4} \bar{u}+a_{5} \frac{d \bar{N}}{d r}+a_{6} \frac{d \bar{\phi}}{d r} \\
\frac{d^{2}}{d r^{2}}\left(\frac{d \bar{\phi}}{d r}\right)+\frac{2}{r} \frac{d}{d r}\left(\frac{d \bar{\phi}}{d r}\right)-\frac{2}{r^{2}}\left(\frac{d \bar{\phi}}{d r}\right)=a_{7} \bar{u}+a_{8} \frac{d \bar{N}}{d r}+a_{9} \frac{d \bar{\phi}}{d r}
\end{gathered}
$$

where

$$
\begin{gathered}
a_{1}=\left(s^{2}-n_{2} a_{7} \frac{a}{s^{2}}\right), a_{2}=\left(n_{1}-n_{2} a_{8} \frac{a}{s^{2}}\right), a_{3}=n_{2}\left(1-\frac{a}{s^{2}} a_{9}\right) \\
a_{4}=\frac{n_{4}}{\tau} \frac{a}{s^{2}} a_{7}, a_{5}=\left(n_{3}\left(s+\frac{1}{\tau}\right)+\frac{n_{4}}{\tau} \frac{a}{s^{2}} a_{8}\right), a_{6}=\frac{n_{4}}{\tau}\left(\frac{a}{s^{2}} a_{9}-1\right) \\
a_{7}=\frac{n_{6} s^{3}}{\left(1+\frac{a}{s}\left(1+n_{6} n_{2}\right)\right)}, a_{8}=\frac{\left(s n_{6} n_{1}-\frac{n_{5}}{\tau}\right)}{\left(1+\frac{a}{s}\left(1+n_{6} n_{2}\right)\right)}, a_{9}=\frac{s\left(1+n_{6} n_{2}\right)}{\left(1+\frac{a}{s}\left(1+n_{6} n_{2}\right)\right)} .
\end{gathered}
$$

Now, it is possible to get the solutions of the coupled system of differential Equations (30)-(32) by the eigenvalues method proposed in [30-34]. From Equations (30)-(32), the matrix-vectors can be expressed in the form

$$
L V=A V,
$$

where $L=\frac{d^{2}}{d r^{2}}+\frac{2}{r} \frac{d}{d r}-\frac{2}{r^{2}}, V=\left[\begin{array}{lll}\bar{u} & \frac{d \bar{N}}{d r} & \frac{d \bar{\phi}}{d r}\end{array}\right]^{T}$ and $A=\left[\begin{array}{ccc}a_{1} & a_{2} & a_{3} \\ a_{4} & a_{5} & a_{6} \\ a_{7} & a_{8} & a_{9}\end{array}\right]$.

The characteristic equation of matrix $A$ can be expressed as

$$
\begin{gathered}
\omega^{3}-\omega^{2}\left(a_{5}+a_{9}+a_{1}\right)+\omega\left(a_{5} a_{9}-a_{9} a_{5}+a_{5} a_{1}+a_{9} a_{1}-a_{4} a_{2}-a_{7} a_{3}\right)+a_{6} a_{8} a_{1}- \\
a_{5} a_{9} a_{1}-a_{6} a_{7} a_{2}+a_{4} a_{9} a_{2}+a_{5} a_{7} a_{3}-a_{4} a_{8} a_{3}=0 .
\end{gathered}
$$

The eigenvalues of matrix $A$ are the three roots of Equation (34) which are defined by the form $s \omega_{1}, \omega_{2}, \omega_{3}$. Thus, the corresponding eigenvectors $Y$ can be computed by:

$$
Y=\left(\begin{array}{c}
-a_{6} a_{2}+\left(-\omega+a_{5}\right) a_{3} \\
a_{6}\left(-\omega+a_{1}\right)-a_{4} a_{3} \\
-\omega^{2}+a_{5}\left(\omega-a_{1}\right)+\omega a_{1}+a_{4} a_{2}
\end{array}\right) .
$$

The solutions of Equation (33) which are bounded as $r \rightarrow \infty$ can be given by

$$
\mathrm{V}(r, s)=\sum_{i=1}^{3} A_{i} Y_{i} X_{i} r^{-1 / 2} K_{3 / 2}\left(m_{i} r\right),
$$

where $m_{i}=\sqrt{\omega_{i}}, R_{1}, R_{2}$ and $R_{3}$ are constants that can be determined by using the boundary conditions of the problem and $K_{3 / 2}$ is the modification of Bessel's function of order $\frac{3}{2}$. Thus, the solutions of variables with respect to $r$ and $s$ can take the forms:

$$
\begin{aligned}
\bar{u}(r, s) & =\sum_{i=1}^{3} A_{i} U_{i} r^{-1 / 2} K_{3 / 2}\left(m_{i} r\right), \\
\bar{N}(r, s) & =-\sum_{i=1}^{3} A_{i} N_{i} r^{-1 / 2} K_{1 / 2}\left(m_{i} r\right), \\
\bar{\phi}(r, s) & =-\sum_{i=1}^{3} A_{i} T_{i} r^{-1 / 2} K_{1 / 2}\left(m_{i} r\right),
\end{aligned}
$$




$$
\begin{gathered}
\bar{T}(r, s)=-\sum_{i=1}^{3} A_{i} \frac{T_{i}\left(1-\frac{a}{s^{2}} m_{i}^{2}\right)}{r^{1 / 2} m_{i}} K_{1 / 2}\left(m_{i} r\right), \\
\bar{\sigma}_{r r}(r, s)=\sum_{i=1}^{3} A_{i}\left(\frac{n_{1} N_{i}+\left(1-\frac{a}{s^{2}} m_{i}^{2}\right) n_{2} T_{i}-m_{i}^{2} U_{i}}{r^{1 / 2} m_{i}} K_{1 / 2}\left(m_{i} r\right)-\frac{2\left(1-n_{7}\right) U_{i}}{r^{3 / 2}} K_{3 / 2}\left(m_{i} r\right)\right) \\
\bar{\sigma}_{\theta \theta}(r, s)=\bar{\sigma}_{\phi \phi}(r, s)=\sum_{i=1}^{3} A_{i}\left(\frac{n_{1} N_{i}+\left(1-\frac{a}{s^{2}} m_{i}^{2}\right) n_{2} T_{i}-m_{i}^{2} n_{7} U_{i}}{r^{1 / 2} m_{i}} K_{1 / 2}\left(m_{i} r\right)+\frac{\left(1-n_{7}\right) U_{i}}{r^{3 / 2}} K_{3 / 2}\left(m_{i} r\right)\right) .
\end{gathered}
$$

\section{Numerical Results and Discussions}

Now, we consider a numerical example; for computational purposes, a silicon (Si) like material has been considered. Assuming that an isotropic semiconductor, silicon-like material with the physical constants that are listed below [35]:

$$
\begin{gathered}
\rho=2330(\mathrm{~kg})\left(\mathrm{m}^{-3}\right), c_{e}=695(\mathrm{~J})\left(\mathrm{kg}^{-1}\right)\left(\mathrm{k}^{-1}\right), E_{g}=1.11(\mathrm{eV}), t_{p}=1.2, \\
\lambda=3.64 \times 10^{10}(\mathrm{~N})\left(\mathrm{m}^{-2}\right), d_{n}=-9 \times 10^{-31}\left(\mathrm{~m}^{3}\right), \alpha_{t}=3 \times 10^{-6}\left(\mathrm{k}^{-1}\right), \\
\mu=5.46 \times 10^{10}(\mathrm{~N})\left(\mathrm{m}^{-2}\right), \tau=5 \times 10^{-5}(\mathrm{~s}), s_{o}=2(\mathrm{~m})\left(\mathrm{s}^{-1}\right), n_{o}=10^{20}\left(\mathrm{~m}^{-3}\right), \\
D_{e}=2.510^{-3}\left(\mathrm{~m}^{2}\right)\left(\mathrm{s}^{-1}\right), T_{o}=300(\mathrm{k}), t=2, a=0.3 .
\end{gathered}
$$

A numerical inversion method adopted the general solutions of the thermodynamic and conductive temperatures, the carrier density, displacement, and the stress distributions; Stehfest's [36] numerical inversion method has been chosen. In this method, the Laplace transforms inverse for $\bar{h}(x, y, s)$ are approximated as

$$
\mathrm{h}(x, y, t)=\frac{\ln (2)}{t} \sum_{n=1}^{N} V_{n} \overline{\mathrm{h}}\left(x, y, n \frac{\ln (2)}{t}\right)
$$

where

$$
V_{n}=(-1)^{\left(\frac{N}{2}+1\right)} \sum_{p=\frac{n+1}{2}}^{\min \left(n \frac{N}{2}\right)} \frac{(2 p) ! p^{\left(\frac{N}{2}+1\right)}}{p !(n-p) !\left(\frac{N}{2}-p\right) !(2 n-1) !},
$$

where $N$ is the term numbers. Based on the Laplace transform's inverse and the above set of constants, the calculations of physical variables with respect to the redial distance $r$ under one temperature, classical two-temperature and hyperbolic two-temperature are presented in Figures 1-6. The variation of conductive temperature, the variation of thermodynamic temperature, the variation of radial displacement, the variation of carrier density, and the distributions of radial and hoop stresses along the radial distance $r$ under the coupled photothermal theories are calculated numerically. Figure 1 displays the variations of conductive temperature versus the radial distance $r$. It is clear that the conductive temperature has maximum value at the boundary $r=1$, after that it progressively decreases with the increasing of the radial distance $r$, to eventually come to zero. Figure 2 shows the thermodynamic temperature variations versus the radial distance $r$. It is observed that it starts from the highest values at the boundary $r=1$ and decreases with the increasing of the radial distance $r$ until it reaches zero for sufficiently large values of $r$. Figure 3 explains the carrier density variations with respect to the radial distance $r$. It is clear that the density of the carrier begins with its highest values at the boundary $r=1$, then it progressively decreases with the increasing of the redial distance $r$ until it comes to zero. Figure 4 depicts the variations of radial displacement versus the radial distance $r$. It can be observed that the displacement attains a maximum negative value then it increases progressively up to peak values in a particular location proximately close to the surface, and then decreases to zero. Figure 5 shows the variations of radial stress with respect to the redial distance $r$. It is noticed that the radial stress, permanently begins from zero and drops to zero to obey the boundary conditions. 
The hoop stress variations versus the radial distance $r$ is shown in Figure 6. It can be noticed that the hoop stress attains a maximum negative value and then continuously increases to zero. Finally, in compression between the solutions, one can conclude that considering the coupled photothermal theory with thermal and conductive temperatures is an important phenomenon and has a great effect on the distribution of field quantities.

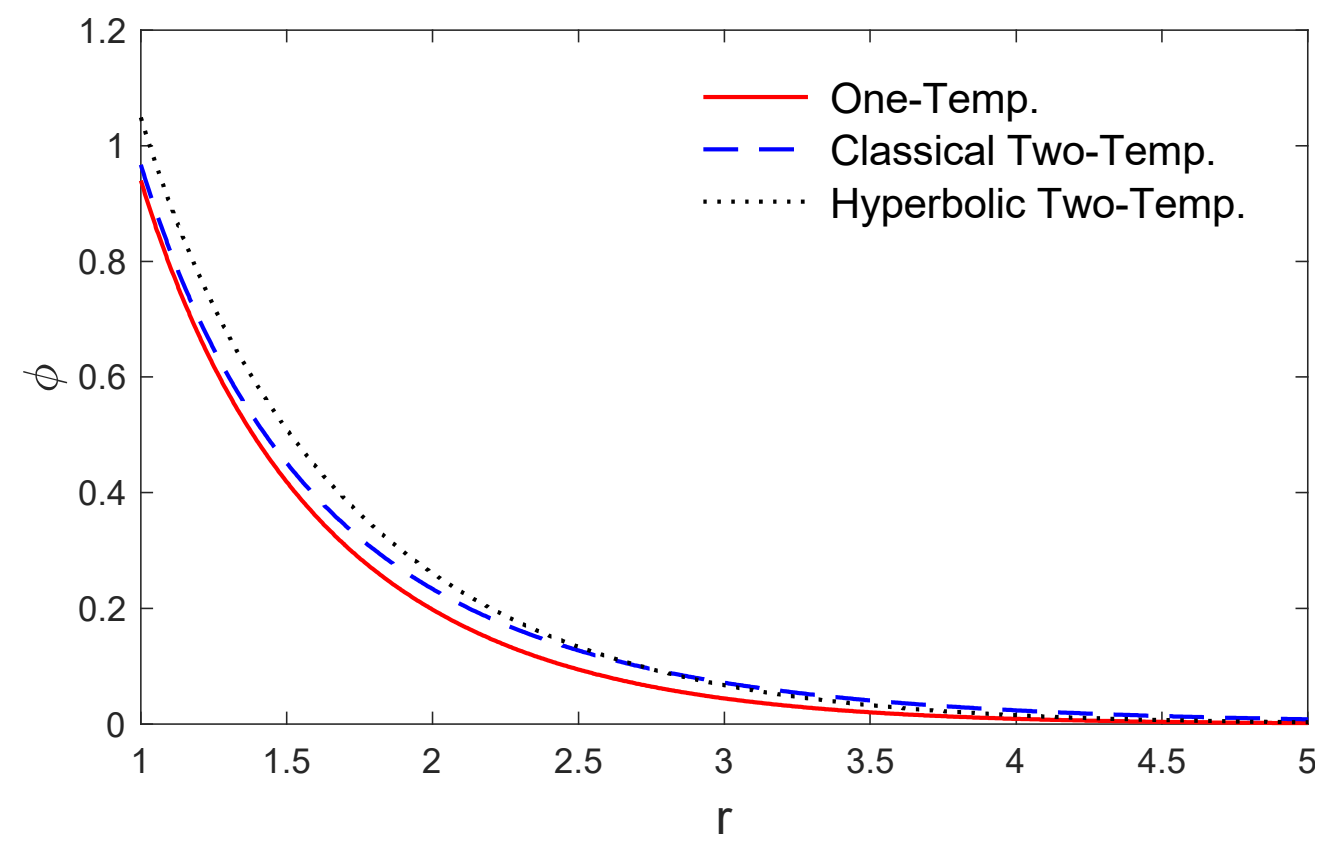

Figure 1. The variation of conductive temperature with distance for different models.

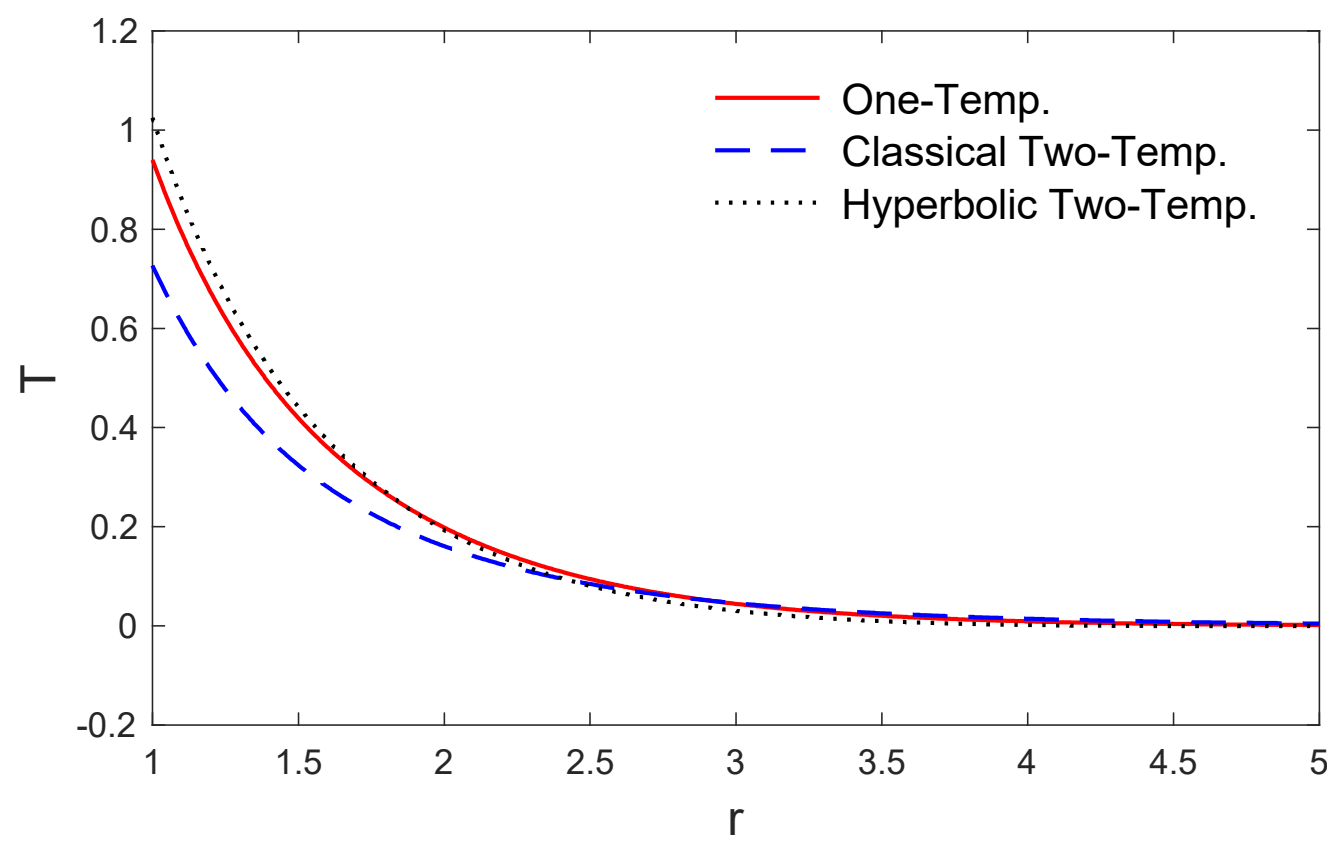

Figure 2. The variation of thermodynamic temperature with distance for different models. 


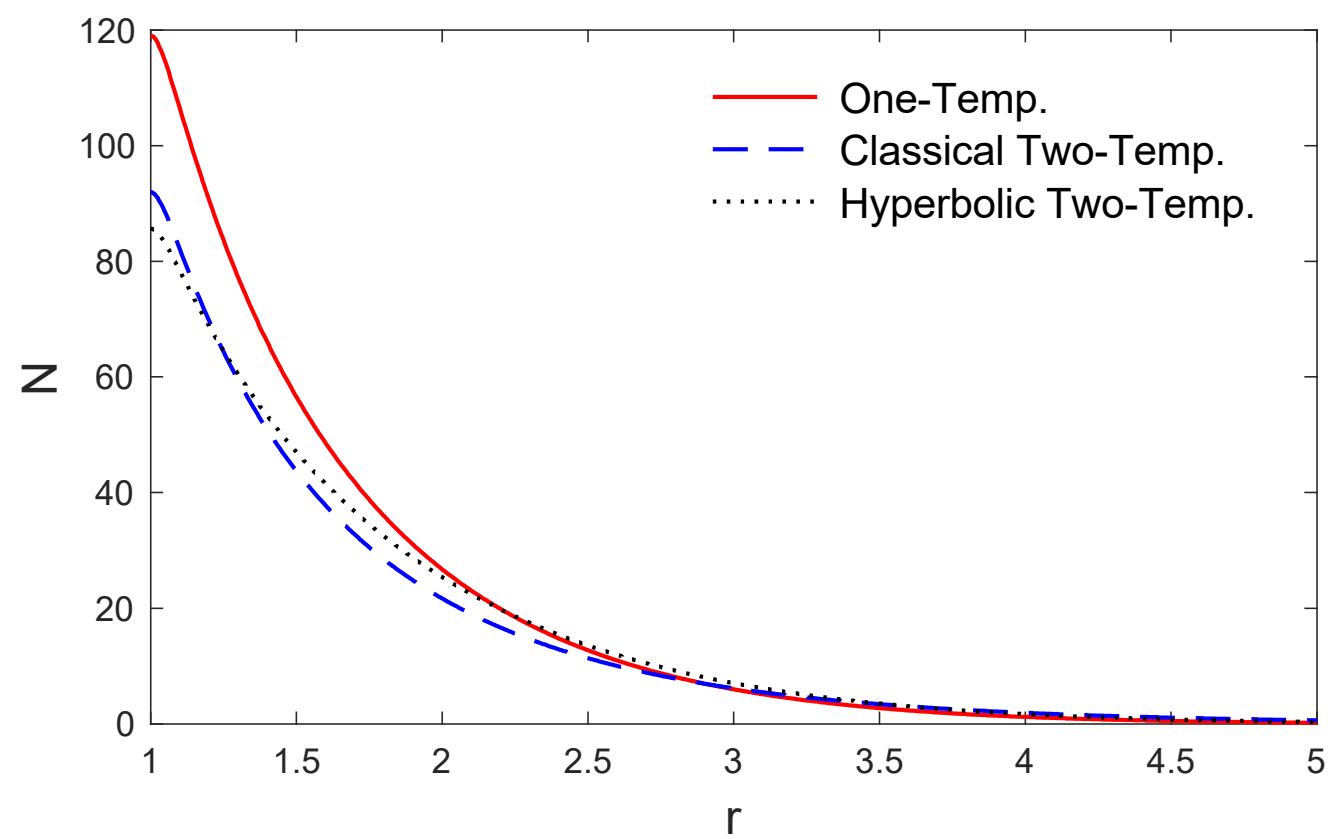

Figure 3. The variation of carrier density with distance for different models.

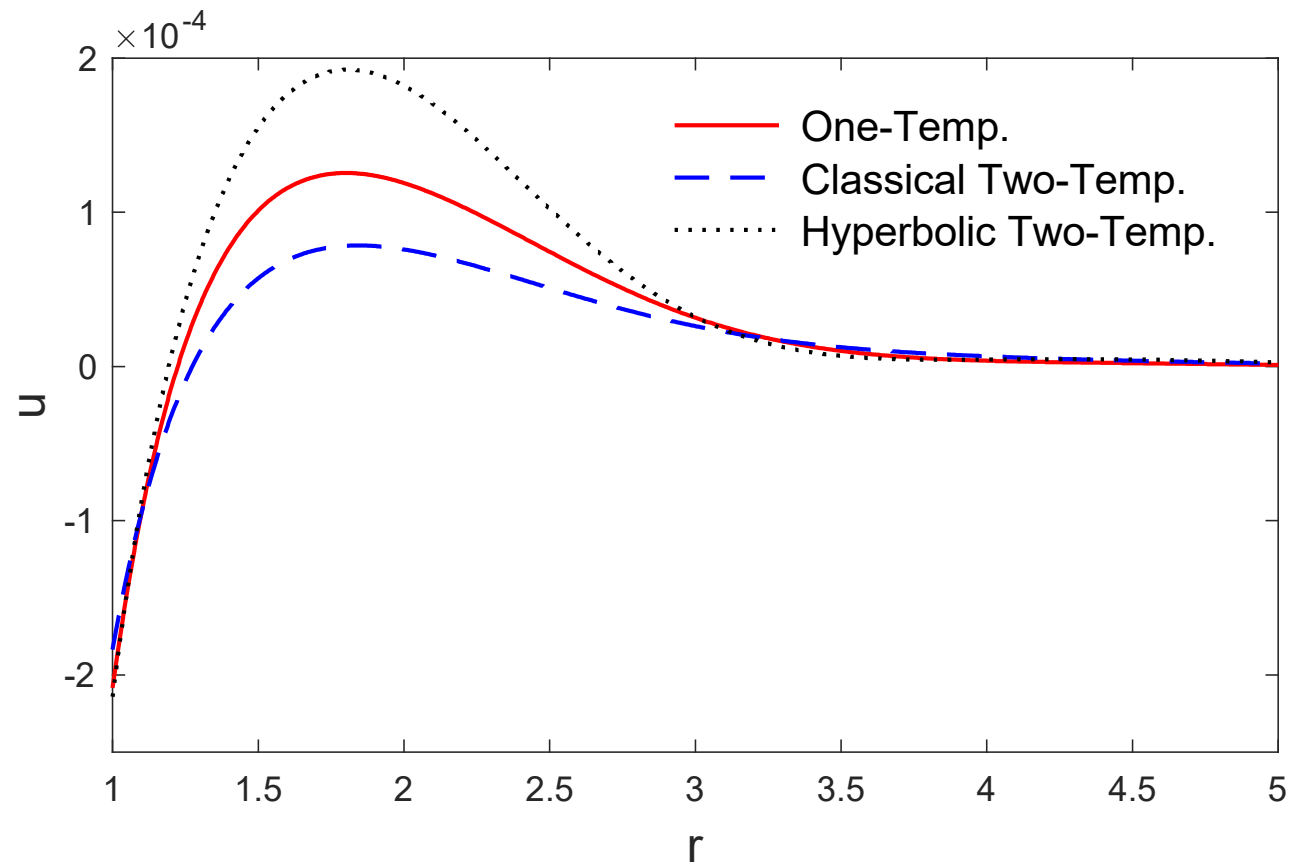

Figure 4. The variation of displacement with distance for different models. 


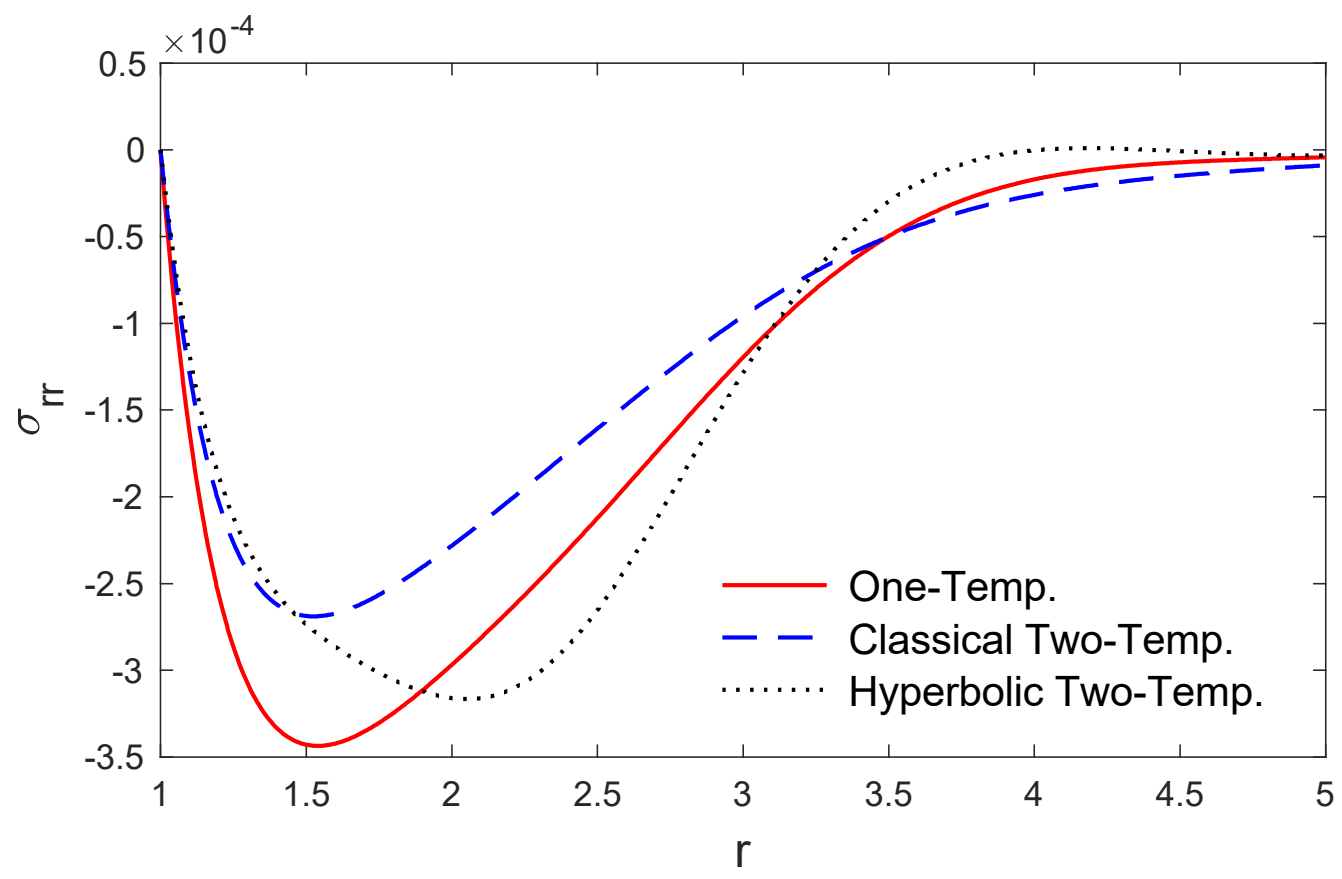

Figure 5. The variation of radial stress with distance for different models.

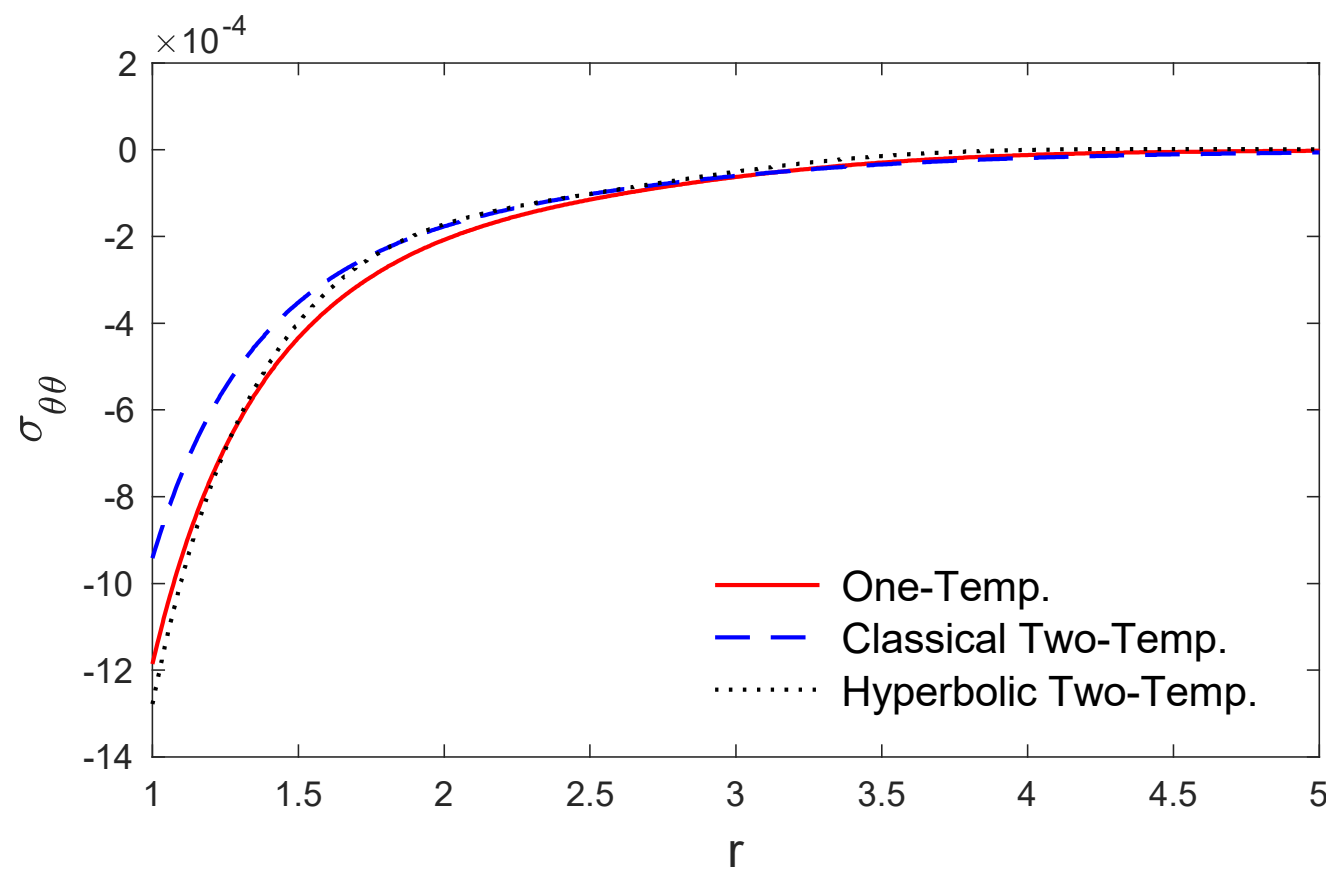

Figure 6. The variation of hoop stress with distance for different models.

\section{Conclusions}

Based on the new hyperbolic two-temperature model of thermoelasticity, the variations of the conductive and the thermodynamic temperatures, the variation of radial displacement, and the variation of carrier density, the distributions of radial and hoop stresses in a semiconductor medium are studied. The non-dimensional result has been solved, employing the Laplace transform and the eigenvalues approach. In comparing the solutions, one can conclude that the coupled photo-thermoelastic theory with hyperbolic thermal and conductive temperatures is an important phenomenon and has important effects on the distribution of field quantities. We believe that the analysis of the present study will be useful to understand the basic features of this new model for heat conduction. 
Author Contributions: All four authors conceived the framework and structured the whole manuscript, checked the results, and completed the revision of the paper. The authors have equally contributed to the elaboration of this manuscript. All authors have read and agreed to the published version of the manuscript.

Funding: This project was funded by the Deanship of Scientific Research (DSR) at King Abdulaziz University, Jeddah, Saudi Arabia, under grant no. (KEP-92-130-38). The authors, therefore, acknowledge with thanks DSR technical and financial support.

Conflicts of Interest: The authors declare no conflicts of interest.

\section{References}

1. Gurtin, M.E.; Williams, W.O. An axiomatic foundation for continuum thermodynamics. Arch. Ration. Mech. Anal. 1967, 26, 83-117. [CrossRef]

2. Chen, P.J.; Gurtin, M.E. On a theory of heat conduction involving two temperatures. Z. für Angew. Math. und Phys. 1968, 19, 614-627. [CrossRef]

3. Chen, P.J.; Gurtin, M.E.; Williams, W.O. On the thermodynamics of non-simple elastic materials with two temperatures. Z. für Angew. Math. und Phys. 1969, 20, 107-112. [CrossRef]

4. Ezzat, M.A.; El-Karamany, A.; Ezzat, S.M. Two-temperature theory in magneto-thermoelasticity with fractional order dual-phase-lag heat transfer. Nucl. Eng. Des. 2012, 252, 267-277. [CrossRef]

5. Ezzat, M.A.; Youssef, H.M. Two-Temperature Theory in Three-Dimensional Problem for Thermoelastic Half Space Subjected to Ramp Type Heating. Mech. Adv. Mater. Struct. 2013, 21, 293-304. [CrossRef]

6. Deswal, S.; Kalkal, K. Two Temperature Magneto-Thermoelasticity with Initial Stress: State Space Formulation. J. Thermodyn. 2013, 2013, 1-12. [CrossRef]

7. Abbas, I.; Kumar, R.; Reen, L.S. Response of thermal source in transversely isotropic thermoelastic materials without energy dissipation and with two temperatures. Can. J. Phys. 2014, 92, 1305-1311. [CrossRef]

8. Youssef, H.M.; El-Bary, A.A. Theory of hyperbolic two-temperature generalized thermoelasticity. Mater. Phys. Mech. 2018, 40, 158-171.

9. Marin, M.; Vlase, S.; Ellahi, R.; Bhatti, M. On the Partition of Energies for the Backward in Time Problem of Thermoelastic Materials with a Dipolar Structure. Symmetry 2019, 11, 863. [CrossRef]

10. Marin, M.; Ellahi, R.; Chirilă, A. On solutions of Saint-Venant's problem for elastic dipolar bodies with voids. Carpathian J. Math. 2017, 33, 219-232.

11. Todorović, D.M. Plasma, thermal, and elastic waves in semiconductors. Rev. Sci. Instrum. 2003, 74, 582.

12. Todorović, D.M. Photothermal and electronic elastic effects in microelectromechanical structures. Rev. Sci. Instrum. 2003, 74, 578 .

13. Song, Y.; Todorović, D.M.; Cretin, B.; Vairac, P. Study on the generalized thermoelastic vibration of the optically excited semiconducting microcantilevers. Int. J. Solids Struct. 2010, 47, 1871-1875. [CrossRef]

14. Yasein, M.; Mabrouk, N.; Lotfy, K.; El-Bary, A. The influence of variable thermal conductivity of semiconductor elastic medium during photothermal excitation subjected to thermal ramp type. Results Phys. 2019, 15, 102766. [CrossRef]

15. Ailawalia, P.; Kumar, A. Ramp Type Heating in a Semiconductor Medium under Photothermal Theory. Silicon 2019, 12, 347-356. [CrossRef]

16. Abbas, I.; Hobiny, A. Photo-thermal-elastic interaction in an unbounded semiconducting medium with spherical cavity due to pulse heat flux. Waves Random Complex Media 2017, 28, 1-13. [CrossRef]

17. Lotfy, K.; Tantawi, R.S.; Anwer, N. Response of Semiconductor Medium of Variable Thermal Conductivity Due to Laser Pulses with Two-Temperature through Photothermal Process. Silicon 2019, 11, 2719-2730. [CrossRef]

18. Zenkour, A.M.; Abbas, I.A. Nonlinear transient thermal stress analysis of temperature-dependent hollow cylinders using a finite element model. Int. J. Struct. Stab. Dy. 2014, 14, 1450025. [CrossRef]

19. Mohamed, R.; Abbas, I.; Abo-Dahab, S. Finite element analysis of hydromagnetic flow and heat transfer of a heat generation fluid over a surface embedded in a non-Darcian porous medium in the presence of chemical reaction. Commun. Nonlinear Sci. Numer. Simul. 2009, 14, 1385-1395. [CrossRef]

20. Abbas, I.; El-Amin, M.; Salama, A.; El-Amin, M.F. Effect of thermal dispersion on free convection in a fluid saturated porous medium. Int. J. Heat Fluid Flow 2009, 30, 229-236. [CrossRef] 
21. Zenkour, A.M.; Abbas, I. A generalized thermoelasticity problem of an annular cylinder with temperature-dependent density and material properties. Int. J. Mech. Sci. 2014, 84, 54-60. [CrossRef]

22. Abbas, I.; Zenkour, A.M. The Effect of Rotation and Initial Stress on Thermal Shock Problem for a Fiber-Reinforced Anisotropic Half-Space Using Green-Naghdi Theory. J. Comput. Theor. Nanosci. 2014, 11, 331-338. [CrossRef]

23. Abbas, I.; Kumar, R. Deformation Due to Thermal Source in Micropolar Generalized Thermoelastic Half-Space by Finite Element Method. J. Comput. Theor. Nanosci. 2014, 11, 185-190. [CrossRef]

24. Abbas, I. Nonlinear transient thermal stress analysis of thick-walled FGM cylinder with temperature-dependent material properties. Meccanica 2014, 49, 1697-1708. [CrossRef]

25. Marin, M. Lagrange identity method for microstretch thermoelastic materials. J. Math. Anal. Appl. 2010, 363, 275-286. [CrossRef]

26. Marin, M.; Nicaise, S. Existence and stability results for thermoelastic dipolar bodies with double porosity. Contin. Mech. Thermodyn. 2016, 28, 1645-1657. [CrossRef]

27. Song, Y.; Bai, J.T.; Ren, Z.Y. Study on the reflection of photothermal waves in a semiconducting medium under generalized thermoelastic theory. Acta Mech. 2012, 223, 1545-1557. [CrossRef]

28. Mandelis, A.; Nestoros, M.; Christofides, C. Thermoelectronic-wave coupling in laser photothermal theory of semiconductors at elevated temperatures. Opt. Eng. 1997, 36, 459. [CrossRef]

29. Zenkour, A.M.; Abouelregal, A.E. Effect of temperature dependency on constrained orthotropic unbounded body with a cylindrical cavity due to pulse heat flux. J. Therm. Sci. Technol. 2015, 10, JTST0019. [CrossRef]

30. Das, N.C.; Lahiri, A.; Giri, R.R. Eigenvalue approach to generalized thermoelasticity. Indian J. Pure Appl. Math. 1997, 28, 1573-1594.

31. Abbas, I. Eigenvalue approach in a three-dimensional generalized thermoelastic interactions with temperature-dependent material properties. Comput. Math. Appl. 2014, 68, 2036-2056. [CrossRef]

32. Abbas, I. Eigenvalue approach for an unbounded medium with a spherical cavity based upon two-temperature generalized thermoelastic theory. J. Mech. Sci. Technol. 2014, 28, 4193-4198. [CrossRef]

33. Abbas, I. A Dual Phase Lag Model on Thermoelastic Interaction in an Infinite Fiber-Reinforced Anisotropic Medium with a Circular Hole. Mech. Based Des. Struct. Mach. 2015, 43, 501-513. [CrossRef]

34. Abbas, I. The effects of relaxation times and a moving heat source on a two-temperature generalized thermoelastic thin slim strip. Can. J. Phys. 2015, 93, 585-590. [CrossRef]

35. Song, Y.; Todorović, D.M.; Cretin, B.; Vairac, P.; Xu, J.; Bai, J. Bending of Semiconducting Cantilevers Under Photothermal Excitation. Int. J. Thermophys. 2014, 35, 305-319. [CrossRef]

36. Stehfest, H. Algorithm 368: Numerical inversion of Laplace transforms [D5]. Commun. ACM 1970, 13, 47-49. [CrossRef]

(C) 2020 by the authors. Licensee MDPI, Basel, Switzerland. This article is an open access article distributed under the terms and conditions of the Creative Commons Attribution (CC BY) license (http://creativecommons.org/licenses/by/4.0/). 